\title{
Research on Photoelectric Tracking System Based on Rotating Voice Coil Motor
}

\author{
Xuanlin Huang ${ }^{1,2,3, a}$, Sisi Chen ${ }^{1,2,3}$ and TaoTang ${ }^{1,2}$ \\ ${ }^{1}$ Key Laboratory of Optical Engineering, Chinese Academy of Science, Chengdu, 610209, China \\ 2 Institute of Optics and Electronics, Chinese Academy of Science, Chengdu, 610209, China \\ 3University of Chinese Academy of Science, Beijing, 100049, China \\ Ahuang3xuanlin@163.com \\ ${ }^{*}$ Xuanlin Huang
}

Keywords: ATP, rotating voice coil motor,torque analysis,tracking error.

Abstract. Photoelectric tracking system is widely used in space exploration, manipulation, laser communication and other fields. The implementation of ATP system is the key to achieve tracking. The use of double-closed loop control can give the system a higher stiffness, improve the system's anti-interference ability. The structure of the system is analyzed and the torque analysis of the rotating voice coil motor is carried out. Through the identification of the system function, the system is designed for the closed loop transfer function. The sine tracking experiment followed by a position error of $<1.5$ ', and a double-axis maneuvering experiment with a closed loop of CCD was performed with an average tracking error of $<2$ pixels. The results show that the ATP coarse tracking mechanism driven by the rotating voice coil motor can achieve high tracking accuracy under the control structure of single sensor with double-closed loop.And the rotating voice coil motor with high structure density, but also a single sensor makes the system smaller.

\section{Introduction}

The tracking system ——acquisition,tracking,pointing process (referred to as ATP) generally uses the compound axis structure to track the target, and the actuator of the coarse tracking mechanism of this system adopts the rotating voice coil motor. ATP system in the two-dimensional pendulum mirror structure similar to the turntable, from the azimuth, pitch two dimensions of the drive and two-dimensional turntable structure similar. Many countries space optical communication project ATP system is also used to drive turntables such as ESLEX SILEX space optical communication plan, Japan OICETS satellite equipped with laser communication device[1][2].

The latest voice coil motor control strategy is generally a combination of modern control and PID: [3] proposed a motor based on adaptive fuzzy PID control program, the method largely eliminates the inaccurate modeling and load changes caused by Disturbance. In [4], a nonlinear PID strategy is proposed. The two-degree-of-freedom structure proposed in [5] includes one for the disturbance observer (DOB) and the other for the adaptive robust control (ARC), which shows better performance than the traditional mode switching servo system. The above control strategy can effectively improve the corresponding indicators, but there are some problems, high computational complexity is difficult to achieve real-time requirements of the ATP system, and some methods of dependence on the model high system load changes when the results It is very different

\section{Principle and analysis of rotating voice coil motor}

\subsection{Principle of rotating voice coil motor}

The rotating voice coil motor is a special form of direct drive motor based on the principle of amber force[6]. The ends of the motor are bent into arcs, and the energizing coils are subjected to the effect of the ammeter in the magnetic field to form the moment. Rotating the voice coil motor travel path is 
curved, which makes the motor more compact in structure, can make the system smaller. The electrical part of the rotating voice coil motor can be regarded as an L-R network, resulting in the following electromotive force balance equation(1-2)whose motor armature terminal voltage is $u_{a}$, back electromotive force is $e_{a}$, air gap magnetic density average is $B_{g}$,coil effective length is $L_{e}$, the torque balance equation(3)whose motor torque coefficient is $k_{a}$, angular velocity is $w$, total moment is $T$, moment of inertia is $T_{m}$, friction torque is $T_{c}$, kinematic moment of inertia is $J$, friction damping coefficient is $k$, force arm length is $r$ :

$$
\begin{gathered}
u_{a}=e_{a}+i_{a} R_{a}+L_{a} \frac{d i_{a}}{d t} \\
e_{a}=B_{g} L_{e} \cdot w=k_{a} w \\
T=B_{g} L_{e} i_{a} r=T_{m}+T_{c}=J \frac{d w}{d t}+k w
\end{gathered}
$$

According to the above-mentioned torque balance equation, the voltage balance equation, the simultaneous and the Lagrangian transformation to obtain the armature terminal voltage and position transfer function.

$$
G(s)=\frac{k_{a}}{L_{a} J s^{3}+\left(L_{a} k+R_{a} J\right) s^{2}+\left(R_{a} k+k_{a}^{2}\right) s+1}
$$

The double-axis open-loop transfer function is measured by sweeping method, and the double axis velocity open-loop approximation model is obtained.

$$
\begin{aligned}
G_{E} & =\frac{5}{6.333 \times 10^{-3} s^{2}+0.2292 s+1} \cdot \frac{s}{31.83 s+1} \\
G_{A} & =\frac{2}{4.503 \times 10^{-4} s^{2}+0.06069 s+1} \cdot \frac{s}{6.366 s+1}
\end{aligned}
$$

\subsection{System torque analysis}

Two-dimensional pendulum mechanism using the classic two-dimensional turntable form. Pendulum mirror with quartz material, behind the weight loss design, pendulum mirror back and backplane connection, backplane and U-shaped frame elevation axis connected to the back material selection of steel, with the mirror material thermal expansion coefficient match, Pendulum mirror and U-shaped frame material caused by different thermal deformation. The two-dimensional turntable uses the encoder to measure the deflection angle directly, which achieves higher angular accuracy and control bandwidth. The selected torch voice coil torque is $0.22 \mathrm{Nm}$. Encoder adopt resolution 18 bit, accuracy \pm 15 . "Tilt shaft with one end fixed, one end of the way, fixed end with a pair of angular contact bearing 7202 (back to back way), swimming one end with a deep groove ball bearings 6202, This is done to prevent the shaft from being stuck due to thermal deformation. The bearing is mounted on the housing with a pair of angular contact bearings 7206 (back to back)[7]. It is analyzed whether the rotating voice coil motor can provide the required torque for the whole system and whether there is enough torque margin to realize the closed-loop control with higher bandwidth, and estimate the starting torque of the azimuth and pitch axis respectively. The starting torque of the pitching shaft, including the inertia moment and friction torque of the two parts. The maximum angular acceleration is $\varepsilon$, the coefficient of friction is $\mu$, the equivalent load of the bearing is $P$, the inner diameter of the bearing is $d$, and the safety factor is $\mathrm{S}$ which we use 2 .

$$
\begin{gathered}
T_{s}=\left(T_{m}+T_{f}\right) S \\
T_{m}=J_{E} \varepsilon
\end{gathered}
$$




$$
T_{f}=0.5 \mu P d
$$

According to the parameters of the system can be estimated on the pitch axis of the starting torque of $0.0007 \mathrm{Nm}$, the same can also be calculated $0.0018 \mathrm{Nm}$. So the choice of rotating voice coil motor can provide starting torque, there are enough torque margin.

\section{Experiment and analysis}

Closed loop testing of double-axis of two-dimensional steering mirrors. According to the previous analysis, the test experiments include double-axis position tracking error, double-axis velocity tracking error, real-time tracking through the CCD target.

Fig. 1 and Fig. 2 depict the tracking accuracy of a system based on trajectory guided by a sinusoidal curve based on the positional tracking error of the double-axis position. It can be seen that the tracking track is sinusoidal and the maximum position error of the E axis is $0.0185^{\circ}$ at the $500 \mathrm{~Hz}$ sampling frequency and the maximum position error of the A axis is $0.0191^{\circ}$. So when the given amplitude is $6.5^{\circ}$, the tracking error of the system is about 1 '.

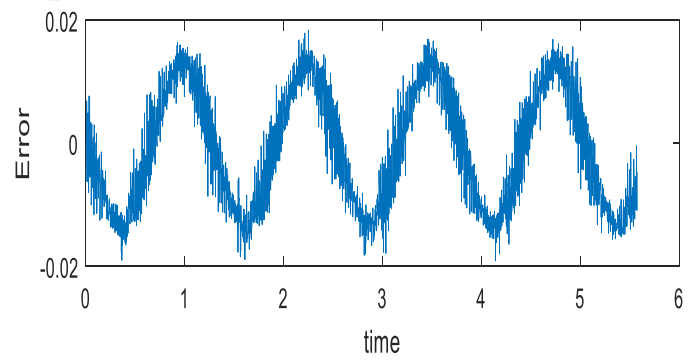

Fig.1 A axis position tracking curve

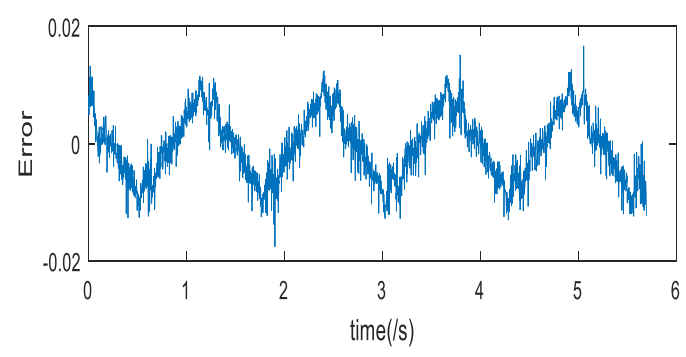

Fig.2 E axis position tracking curve

According to the position error of the E-axis, it is shown that the error is more intense when the commutation is given. The main reason is that the load is large and the gravity is applied to the pitch axis. So the results of the above experiment, which can explain the A-axis position tracking error does not appear such a jagged, in the commutation or can be a good track on the given signal.

Fig. 3 depicts the position of the double-axis position when the target is tracked in real time. When the target is tracked, the closed loop miss range is within 4 pixels. Fig. 4 depicts a description of the system accuracy by the double-axis miss distance at the pixel point $(523,512)$ in the closed-loop of the CCD TV. The maximum miss range of the A axis is 4 pixels, and the maximum miss range of the $\mathrm{E}$ axis is 2 pixels.
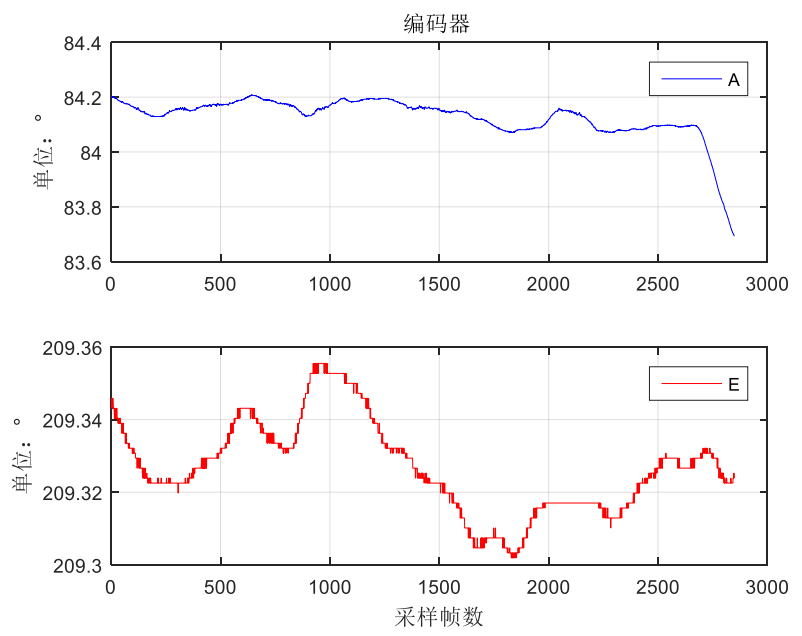

Fig. 3 double-axis real-time tracking trajectory 

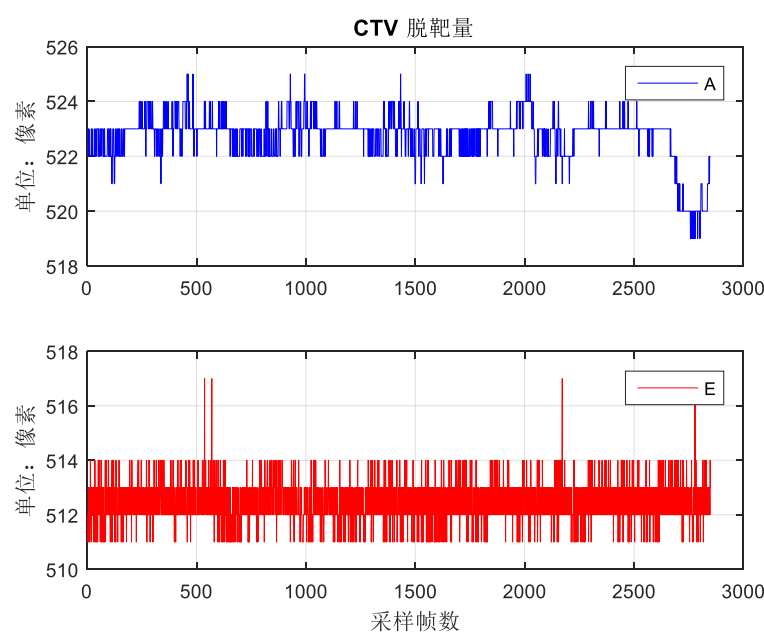

Fig. 4 double-axis missing distance

\section{Summary}

According to the high positioning precision of the rotary voice coil motor, this paper tries to apply it to the photoelectric tracking system, and realizes the control of the large load and reduces the encoder accuracy through the double closed loop PID control strategy. At the same time, only one sensor is used to realize double closed loop control, which makes the ATP system smaller. The experiment shows that the tracking error of the pendulum mirror is within $0.02^{\circ}$ when tracking the sine curve, and the standard deviation of the speed tracking error is about $0.4^{\circ} / \mathrm{s}$. At the same time, the CCD miss distance is measured at the pixel level of $<2$ pixels for real-time object tracking. These two experiments show that the rotating tone coil motor can be used in the photoelectric tracking system to achieve the control accuracy.

\section{References}

[1] Oppenhauser G. SILEX program status: a major milestone is reached[J]. Proceedings of SPIE The International Society for Optical Engineering, 1997, 2990:2-9.

[2] Jono T, Takayama Y, Sodnik Z. OICETS on-orbit laser communication experiments[C]// Lasers and Applications in Science and Engineering. International Society for Optics and Photonics, 2006.

[3] Yu H C, Chen T C, Liu C S. Adaptive Fuzzy Logic Proportional-Integral-Derivative Control for a Miniature Autofocus Voice Coil Motor Actuator With Retaining Force[J]. Magnetics IEEE Transactions on, 2014, 50(11):1-4.

[4] Guo H, Wang D, Xu J. Research on a High-Frequency Response Direct Drive Valve System Based on Voice Coil Motor[J]. IEEE Transactions on Power Electronics, 2012, 28(5):2483-2492

[5] Yi L, Tomizuka M. Two degree-of-freedom control with adaptive robust control for hard disk servo systems[J]. Mechatronics IEEE/ASME Transactions on, 1999, 4(1):17-24.

[6] XING Lianguo ZHOU Huixing HOU Shulin CAO Rongmin, Research and Application of Voice Coil Motor[J], Micromotors, 2011, 44(8):82-87.

[7] TANG Tao, HUANG Yongmei, FU Chenyu,WANG Qiang. Analysis and Design of Multi-closed Loops Control Mode for Tracking Control System Based on Acceleration Feedback[J]. Opto-Electronic Engineering, 2008, 35(7):1-5. 